\title{
Primary orbital tuberculosis on the lower eyelid with cold abscess
}

\author{
Hyun Sik Yoon ${ }^{1}$, \\ Young Cheon $\mathrm{Na}^{1}$, \\ Hye Mi Lee ${ }^{2}$ \\ ${ }^{1}$ Department of Plastic and \\ Reconstructive Surgery, Wonkwang \\ University Hospital, Iksan; ${ }^{2}$ Leehyemi \\ Plastic Surgery Clinic, Gwangju, Korea
}

\begin{abstract}
Orbital tuberculosis is a rare form of extrapulmonary tuberculosis, even in endemic areas. It may involve the soft tissue, lacrimal gland, periosteum, or bones of the orbital wall. We present a case of orbital tuberculosis on the lower eyelid. An 18-year-old woman with no underlying disease visited our clinic for evaluation of an oval nodule $(1.5 \times 1.2 \mathrm{~cm})$ on the right lower eyelid. Incision and drainage without biopsy was performed 2 months ago in ophthalmology department, but the periorbital mass had deteriorated, as the patient had erythematous swelling, tenderness, and cervical lymphadenopathy. Visual acuity was normal; there were no signs of proptosis, diplopia, or ophthalmoplegia. Computed tomography revealed a small abscess cavity without bony involvement. We performed an excision and biopsy through a percutaneous incision under local anesthesia. Histological examination revealed a granuloma and was diagnosed as orbital tuberculosis. The patient was additionally treated with anti-tuberculosis therapy for 6 months and recovered without complication or recurrence by 7 months. Orbital tuberculosis occurs in patients with or without associated pulmonary tuberculosis, and should be considered as a differential diagnosis in patients with inflammatory orbital disease and an orbital mass. If recurrence occurs despite adequate initial treatment, we recommend an additional examination and excisional biopsy.
\end{abstract}

Keywords: Abscess / Eyelid / Orbit / Tuberculosis

\section{INTRODUCTION}

The incidence of tuberculosis has steadily declined for decades due to the development of effective antimicrobial drugs along with socioeconomic development [1]. However, in the 1980s, the human immunodeficiency virus (HIV) epidemic, homelessness, overcrowding, and immigration led to the resurgence of tuberculosis [2]. In third-world and developing countries, tuberculosis is a major cause of morbidity and mortality [3]. Commonly, the primary focus with tuberculous is on pulmonary sites, but extrapulmonary sites of infection include lymph

Correspondence: Young Cheon Na

Department of Plastic and Reconstructive Surgery, Wonkwang University Hospital, 895 Muwang-ro, Iksan 54538, Korea

E-mail: ycnadr@hanmail.net

This work was supported by Wonkwang University in 2019.

Received July 12, 2019 / Revised August 9, 2019 / Accepted August 11, 2019 nodes and osteoarticular areas. In fact, any organ can be involved [1]. Orbital tuberculosis is a rare form of extrapulmonary tuberculosis, even in endemic areas. It may involve the soft tissue, lacrimal gland, periosteum, or bones of the orbital wall and can extend to adjacent paranasal sinuses or intracranial cavities $[4,5]$. Herein, we present a case of orbital tuberculosis on the lower eyelid with a review of the literature.

\section{CASE REPORT}

An 18-year-old woman with no underlying disease visited our clinic for evaluation of an oval nodule $(1.5 \times 1.2 \mathrm{~cm})$ on the right lower eyelid. The patient reported that the mass had become larger after 1 week in which she engaged in intense exercise. Observation of the mass revealed it to be hard, non-tender, with no erythema (Fig. 1). The patient was diagnosed with an 


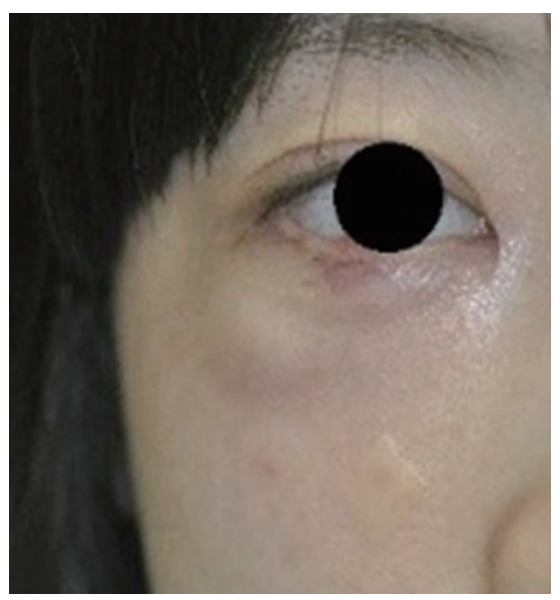

Fig. 1. Photograph of the first ophthalmology department visit. The periorbital mass was hard, non-tender, and with no erythema (this photograph was taken in the ophthalmology department).

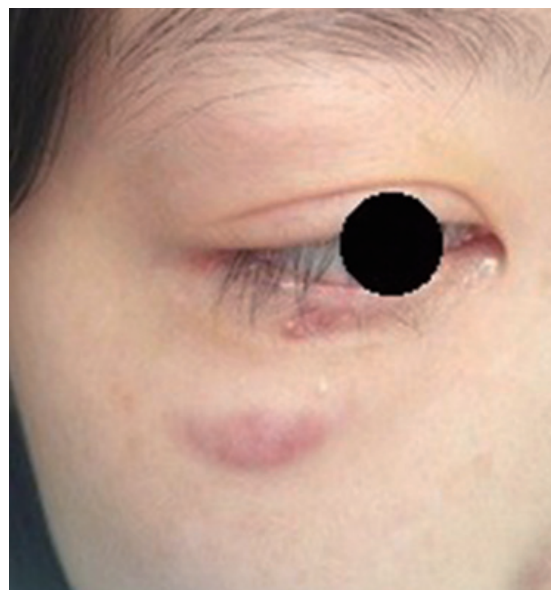

Fig. 2. Photograph of the deteriorated state after ophthalmologic surgery. After incision and drainage without biopsy, the patient's condition was getting worse and he was more aggressive due to repeated erythematous swelling, tenderness symptoms, and cervical lymphadenopathy that persisted for 2 months.

abscess by the ophthalmology department 2 months ago. Under general anesthesia, the abscess was removed through lateral canthotomy and a lateral conjunctival incision without biopsy. Antibiotic treatment was performed. Despite ophthalmologic surgery, the periorbital mass had deteriorated. Symptoms included erythematous swelling, tenderness, and cervical lymphadenopathy (Fig. 2). Visual acuity was normal, and there were no signs of proptosis, diplopia, or ophthalmoplegia. And the patient's general condition was good. On computed tomography, chronic inflammatory change and a small abscess cavity without bony involvement were noted (Fig. 3). We performed an excision and biopsy through a percutaneous incision under local anesthesia. Histological examination revealed a granuloma and was diagnosed as orbital tuberculosis (Fig. 4). There
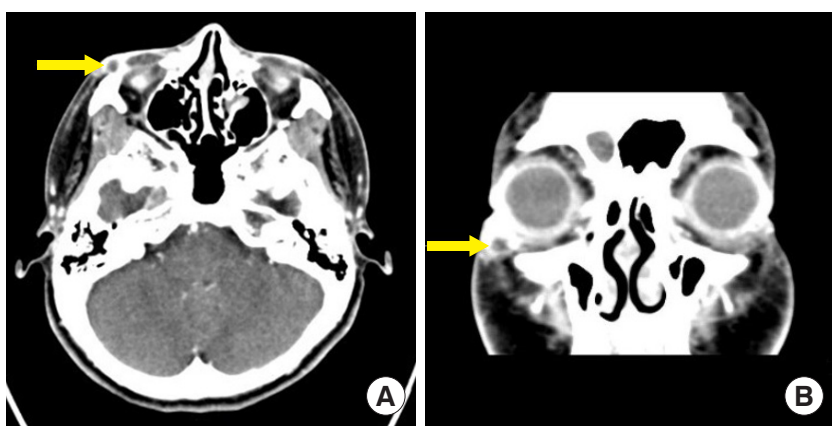

Fig. 3. Computed tomography (CT) findings. (A) Axial view: on CT, chronic inflammatory change and small abscess cavity (arrow) without bony involvement were noted. (B) Coronal view: on CT, chronic inflammatory change and small abscess cavity (arrow) without bony involvement were noted.

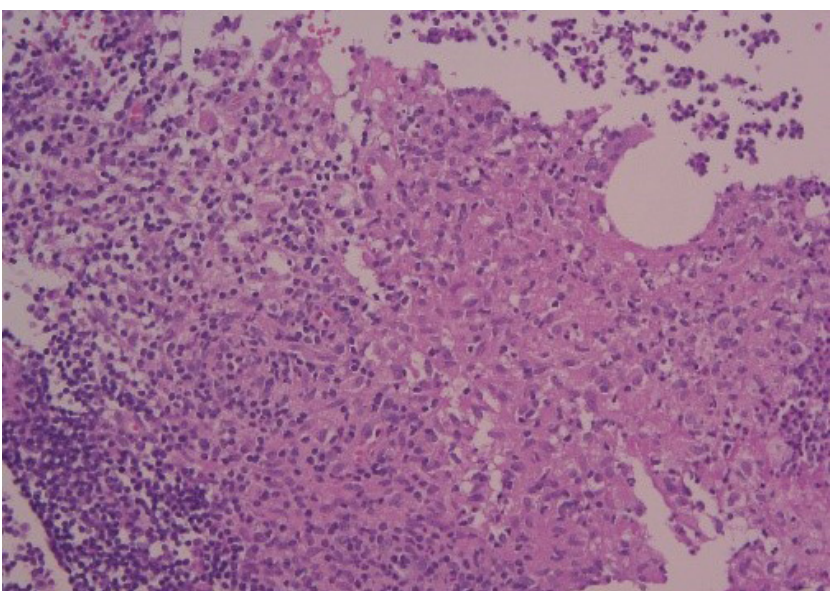

Fig. 4. Histological findings. On histological examination, a granuloma was widely distributed and diagnosed as orbital tuberculosis (H\&E, $\times 200)$.
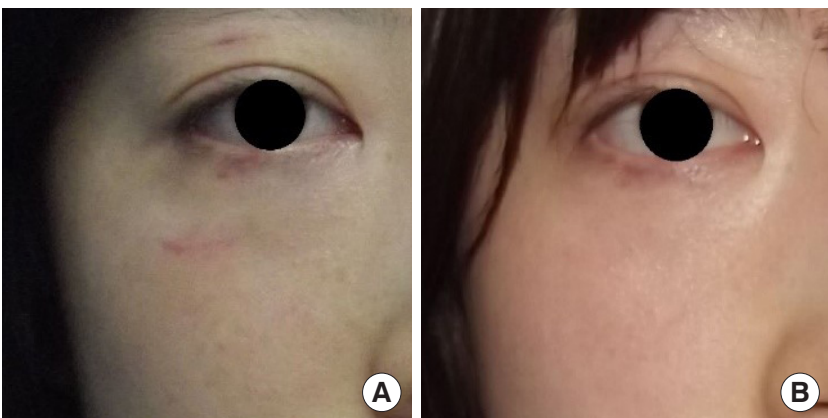

Fig. 5. (A) Photograph at the 3-month follow-up visit. (B) Photograph at the 7-month follow-up visit. The patient had recovered without complication and recurrence.

was no remarkable finding on chest radiography. The patient was treated with anti-tuberculosis therapy (isoniazid, rifampin, pyrazinamide, and ethambutol) for 6 months and recovered without complication or recurrence by the 3- and 7-month follow-up visit (Fig. 5). 
Table 1. Classification of orbital tuberculosis

\begin{tabular}{ll}
\hline Classification & \multicolumn{1}{c}{\begin{tabular}{c} 
Clinical manifestations and radiological findings \\
\hline Classical periostitis
\end{tabular}} \\
$\begin{array}{l}\text { Chronic ulceration, discharging sinus (serosanguinous-purulent). The surrounding skin is } \\
\text { discolored, thickened, and edematous. It may lead to a cicatricial ectropion with consequent } \\
\text { exposure keratitis or prolapse of the palpebral lobe of the lacrimal gland } \\
\text { Bony erosion, irregular thickening of bone, and lytic lesions }\end{array}$ \\
$\begin{array}{ll}\text { Orbital soft tissue tuberculoma or cold abscess without bony destruction } \\
\text { Proptosis, palpable orbital mass lesion, diplopia, and pain } \\
\text { A soft tissue mass, usually isodense to muscle }\end{array}$ \\
$\begin{array}{c}\text { Proptosis, ophthalmoplegia, diplopia, a palpable mass, and reduced vision } \\
\text { Bony destruction, osteomyelitic changes, erosion, and sclerotic changes (the orbital roof or lateral } \\
\text { wall is most frequently involved) }\end{array}$ \\
$\begin{array}{c}\text { Proptosis (most common), globe dystopia, ophthalmoplegia, severe visual loss, conjunctival } \\
\text { congestion, epistaxis, and a contralateral cutaneous-maxillary sinus fistula (the maxillary sinus is } \\
\text { most commonly involved) } \\
\text { Orbital tuberculosis that spread from the paranasal sinuses }\end{array}$ \\
$\begin{array}{c}\text { Limitation of eye movements, ptosis, proptosis, globe displacement, mass in the region of the } \\
\text { lacrimal gland, and regional lymphadenopathy }\end{array}$ \\
\hline
\end{tabular}

\section{DISCUSSION}

Orbital tuberculosis can be classified as primary or secondary depending on origin. Primary orbital tuberculosis is an infection of the ocular region with localized symptoms and without systemic involvement. Secondary orbital tuberculosis involves ocular regions through a hematogenous route from a distant site or extension of neighboring structures such as the paranasal sinuses [6]. Orbital tuwberculosis is more commonly seen in children and girls and is usually unilateral. It is found to be more invasive of the left orbit than of the right orbit [5]. The course of the disease is typically slow and the duration of symptoms ranges from 2 months to 7 years [7]. Orbital tuberculosis is associated with HIV infection/AIDS (acquired immune deficiency syndrome) and autoimmune disease taking the immunosuppressant. And the relapse rate varies from $0 \%$ to $27 \%$ depending on the incidence and control of each country $[1,8]$.

Madge et al. [8] classified orbital tuberculosis into five clinical groups according to characteristic clinical manifestations and radiological findings (Table 1). Very rarely, orbital tuberculosis extending into the cranium through the optic foramen has also been reported. Tuberculosis of the anterior visual pathway can cause visual impairment by either optic neuritis or due to the compressive effect of a space-occupying lesion [9]. Because the optic nerve is close to the adjacent paranasal sinuses, when tuberculosis is spread from the paranasal sinus, the infection can cause optic neuritis and papillitis [10]. In this case, the patient could be considered as having type 2 orbital tuberculosis (orbital soft tissue tuberculoma or cold abscess, without bony destruction) because of a palpable, painful periorbital mass without bony destruction, even though we could not find the primary lesion of orbital tuberculosis.
All patients suspected of having orbital tuberculosis should be investigated with chest radiography and computed tomography to find signs of systemic tuberculosis [8]. Patients with orbital tuberculosis usually have lymphocytosis and elevated erythrocyte sedimentation rate in blood examination [11]. The predominant imaging features of orbital tuberculosis are involvement of the orbital bony wall and lacrimal gland, with a soft tissue inflammatory mass/abscess formation. Bony involvement can be seen in the form of cortical irregularity and destruction [3]. Acid-fast bacilli staining is the method of identifying mycobacteria in tissue sections and smears and it is the most rapid way of detecting these infections. However, tissue detection of fast bacilli may be extremely difficult, and Ziehl-Neelsen staining is often negative [11]. Culture of mycobacteria is considered the most sensitive method for detecting infections; however, it may take weeks to months before organisms can be identified. Particularly where the mycobacterial load is low, false negatives do occur. Biopsy is one method of confirmation. The histological hallmark is the epithelioid granuloma, with Langhans giant cells and caseation necrosis [8]. A diagnosis of orbital tuberculosis as suspected by histopathological examination is confirmed by the demonstration of Mycobacterium tuberculosis DNA by polymerase chain reaction (PCR) [4]. PCR, which is a nucleic acid amplification technique is a rapid technique for tuberculosis diagnosis. This method has high specificity but low sensitivity. Immunohistochemical methods that use mycobacterial antigen MPT64 may increase the specificity of histopathological techniques [8]. The antigen MPB64 is specific for M. tuberculosis. The results indicate its $100 \%$ sensitivity for $M$. tuberculosis DNA, so that an improved diagnosis of tuberculosis is possible by application of nested PCR [12]. Other ancillary methods may also be helpful in diagnosis. Purified protein de- 
rivative (PPD) is a helpful test, but it is not conclusive because a positive PPD does not indicate active disease, but only indicates previous contact with $M$. tuberculosis [11]. In addition, tuberculin skin testing and interferon-based immunological tests, which may also help to establish a diagnosis, are possible [8].

Differential diagnoses are essential for diseases with clinical manifestations similar to orbital tuberculosis. In adults, it is necessary to differentiate orbital tuberculosis from pseudotumor of the orbit, lymphoma, and cavernous hemangioma (which cause proptosis) [7]. In children, it is necessary to differentiate orbital tuberculosis from neuroblastoma (which causes orbital bone destruction), and dacryoadenitis (which is an inflammation of the lacrimal gland that can occur in cases of tuberculosis, syphilis, leprosy, cysticercosis, schistosomiasis, lymphoma, and sarcoid) [3,13].

A 6- to 9-month regimen (2 months of isoniazid, rifampin, pyrazinamide, and ethambutol, followed by 4-7 months of isoniazid and rifampin) is recommended as initial therapy unless the organisms are known or strongly suspected to be resistant to first-line drugs. Extended therapy may also be required for patients with bone and joint tuberculosis, delayed treatment response, or drug resistance. Adjunctive corticosteroids may be useful in patients who have tuberculous meningitis, tuberculous pericarditis, or miliary tuberculosis with refractory hypoxemia. One should consider noncompliance, malabsorption, and drug resistance as possible reasons for delayed or suboptimal response to appropriate therapy. The response to therapy and confirmation are assessed by the clinical examination and resolution of inflammation without any specific testing [1].

Tuberculosis is a significant disease in developing countries and third-world countries. Exceptionally, in Korea, the incidence rate is higher than the rate predicted based on the economic and sanitary level. In Korea, where tuberculosis is endemic, orbital tuberculosis should be considered as a differential diagnosis for orbital masses [14]. Often, if patients do not have any obvious clinical symptoms, periorbital masses are misdiagnosed as pseudotumors and are treated with oral corticosteroids, and it is not until after the formation of a discharge sinus that anti-tuberculosis treatments are usually started. Orbital tuberculosis occurs in patients with or without associated pulmonary tuberculosis $[3,15]$. Therefore, we should have a high level of suspicion in orbital tuberculosis patients with inflammatory orbital disease that includes an orbital mass. We strongly recommend basic tests be performed on suspected patients. Additionally, if recurrence occurs despite adequate initial treatment, we recommend an additional examination and excisional biopsy.

\section{NOTES}

\section{Conflict of interest}

No potential conflict of interest relevant to this article was reported.

\section{Ethical approval}

The study was performed in accordance with the principles of the Declaration of Helsinki. Written informed consent was obtained.

\section{Patient consent}

The patient provided written informed consent for the publication and the use of her images.

\section{ORCID}

Hyun Sik Yoon https://orcid.org/0000-0002-2294-5465

Young Cheon Na https://orcid.org/0000-0003-3136-0351

Hye Mi Lee https://orcid.org/0000-0003-3343-6137

\section{REFERENCES}

1. Golden MP, Vikram HR. Extrapulmonary tuberculosis: an overview. Am Fam Physician 2005;72:1761-8.

2. Pillai S, Malone TJ, Abad JC. Orbital tuberculosis. Ophthalmic Plast Reconstr Surg 1995;11:27-31.

3. Narula MK, Chaudhary V, Baruah D, Kathuria M, Anand R. Pictorial essay: orbital tuberculosis. Indian J Radiol Imaging 2010;20:6-10.

4. Shome D, Honavar SG, Vemuganti GK, Joseph J. Orbital tuberculosis manifesting with enophthalmos and causing a diagnostic dilemma. Ophthalmic Plast Reconstr Surg 2006;22:219-21.

5. Aggarwal D, Suri A, Mahapatra AK. Orbital tuberculosis with abscess. J Neuroophthalmol 2002;22:208-10.

6. Kaur A, Kant S, Bhasker SK. Periorbital tuberculosis. Orbit 2007;26:39-42.

7. Kaur A, Agrawal A. Orbital tuberculosis: an interesting case report. Int Ophthalmol 2005;26:107-9.

8. Madge SN, Prabhakaran VC, Shome D, Kim U, Honavar S, Selva D. Orbital tuberculosis: a review of the literature. Orbit 2008;27:267-77.

9. Dewan T, Sangal K, Premsagar IC, Vashishth S. Orbital tuberculoma extending into the cranium. Ophthalmologica 2006; 220:137-9.

10. Das JC, Singh K, Sharma P, Singla R. Tuberculous osteomyelitis and optic neuritis. Ophthalmic Surg Lasers Imaging 2003;34: 409-12.

11. Oliveira BF, Takay FC, Shida TM, Santo RM, Souza AC Jr, Ma- 
tayoshi S. Orbital tuberculosis diagnosed by immunohistochemistry: case reports. Rev Inst Med Trop Sao Paulo 2004;46: 291-4.

12. Biswas J, Roy Chowdhury B, Krishna Kumar S, Lily Therese K, Madhavan HN. Detection of Mycobacterium tuberculosis by polymerase chain reaction in a case of orbital tuberculosis. Orbit 2001;20:69-74.
13. van Assen S, Lutterman JA. Tuberculous dacryoadenitis: a rare manifestation of tuberculosis. Neth J Med 2002;60:327-9.

14. Sodhi PK, Grover SB, Mehta A, Malik KP, Malik AC. Two cases of orbital tuberculosis in children with unique presentations. Ann Ophthalmol 2004;36:55-8.

15. Khalil M, Lindley S, Matouk E. Tuberculosis of the orbit. Ophthalmology 1985;92:1624-7. 